\title{
User-Experience Development
}

\author{
Aaron Marcus \\ Aaron Marcus and Associates, Inc., 1196 Euclid Avenue, \\ Suite 1F, Berkeley, CA, 94708 USA \\ Aaron.Marcus@AMandA.com
}

\begin{abstract}
Developers worldwide wish to understand user-experience development (UXD). UXD comprises activities in user-centered design of user experience, specifically user-interface development (metaphors, mental models, navigation, interaction, and appearance) that are useful for planning, research, analysis, design, implementation, evaluation, and documentation of products/services across a wide number of platforms. This paper summarizes some key concepts and terms.
\end{abstract}

Keywords: Design, development, management, user interface, user experience.

\section{Introduction}

User-experience development (UXD) is of increasing concern to developers of all computer-based products and services throughout the world. In discussing issues of UXD, it is useful to establish a basic glossary. The following terminology is not universal, but is a candidate for canonical, non-overlapping concepts that are used throughout the UXD community.

First of all, what is user-centered design (UCD)? In an earlier publication, the author wrote the following (Marcus, 2004) about UCD:

\section{User-Centered Design and User-Interface Terminology}

Typical steps in the UCD process are the following:

- Plan project

- Analyze needs

- Gather requirements

- Design initial solution

- Evaluate design solutions (iterate with initial and revised design steps)

- Design revised solution

- Evaluate design concepts (iterative)

- Deploy product/service

- Evaluate product/service (iterative)

- Determine future requirements/enhancements

- Maintain and improve processes

- Assess project

M. Kurosu (Ed.): Human Centered Design, HCII 2009, LNCS 5619, pp. 611-617, 2009.

(C) Springer-Verlag Berlin Heidelberg 2009 
More specifically, user-centered development methods include the following techniques used in initial, intermediate, and final stages of development. Many of these, in turn, are the subjects of books focusing on one or more specific techniques:

- Card sorting

- Cognitive walkthroughs

- Design patterns

- Evaluation workshops

- Expert evaluation

- Field studies

- Focus groups

- Guidelines

- Heuristic evaluation

- Interviews

- Rapid and advanced prototyping

- Storyboarding

- Surveys

- Task analysis

- Use cases

- Use scenarios

- User profiles or personas

- User tests

- Workshops

Factors affecting which techniques are used may vary among the following:

- Availability of appropriate, suitable technology

- Availability of equipment, such as cameras or eye-tracking equipment

- Availability of other physical resources, such as testing rooms

- Availability of usability professionals

- Availability of users

- Budget

- Calendar schedule

- Cost of specialized skills, equipment, or tasks

- Experience of the team with different UCD methods

- Size of the project

The effects of user-centered design can be evaluated by criteria such as these:

- Customer satisfaction

- End-user engagement

- Impact on development process

- Improvements over benchmarks

- Increase of usability awareness in the development team.

- New understanding of users, their tasks, or contexts of user

- Project leader satisfaction

- Return on investment (ROI), as measured by metrics established at the beginning 
Suggestions for new design

- Team satisfaction

- Usability of the developed system

- Usability problems identified

The key terms (verbs) of the user-experience development process may be considered to be as follows:

- Planning

- Research

- Analysis

- Design

- Implementation

- Evaluation

- Documentation

- Training

- Maintenance

UXD methodology involves analyzing how users of interactive systems accomplish tasks, and based on this analysis, developing designs that display and communicate information effectively. Objectives include enhancing ease-of-learning, ease-of-use, productivity, brand loyalty, and satisfaction. UIs may be said to consist of the following UI components:

- Metaphors: Easy recognition of terms, images, and concepts

- Mental Model: Appropriate organization and representation of data, functions, tasks, and roles

- Navigation: Efficient movement within the mental model through menus, dialogue boxes, and control panels

- Interaction: Effective input/output sequencing, including feedback

- Appearance: Quality perceptual characteristics (visual, verbal, acoustic, tactile/haptic, even olfactory).

\section{UXD Around the World}

UXD is growing rapidly as an activity throughout the world. Magazines such as User Experience, the member publication of the Usability Professionals Association (http:// www.usabilityprofessionals.org), are devoted to the topic. Organizations have sprung up internationally, like the Human-Centered Design Network (http://www.hcdnet.org) in Japan, that foster growing awareness of "human-centered" design processes centered around the human experience of software-based technology. Major conferences in human-computer interaction (HCI), computer-human interaction (CHI), and userinterface (UI) design, and interaction design all feature sessions, if not tracks, in their programs devoted to UX and UXD. Examples include the conferences such as those of the following organization: 
- ACM/SIGCHI (http://www.sigchi.org)

- American Institute of Graphic Arts (the term used is "experience design") (http://www.aiga.org)

- HCI International (http://www.hcii2009.org/)

- Society of Technical Communication (http://www.stc.org)

- Usability Professionals Association (http://www.usabilityprofessionals.org)

In addition, hundreds of specialized conferences about product/service development for many different platforms, such as mobile devices, Web 2.0, vehicle systems, etc., and specialized contents, such as animation, Web searching, virtual reality/communities, social networking, commerce, games, healthcare, travel, etc., all have sessions and tracks devoted to the topics of UXD.

In the USA, UXnet (http://www.uxnet.org) has arisen to promote communication and interaction among all organizations and professionals involved with user-experience development. Their Website, unusual among some publications, organizations, and companies, makes an effort to define "user experience:" Although this definition may be debated, it begins to provide a focus.

User Experience (abbreviated: UX) is the quality of experience a person has when interacting with a specific design. This can range from a specific artifact, such as a cup, toy or website, up to larger, integrated experiences such as a museum or an airport.

Many enterprises have made significant advances in shifting their attention to UXD. IBM has gained significant fame over the last 5-10 years for its ease of use Website. The author has also viewed the internal intranet available to IBM staff that provides an archive of documents about specifications, processes, resources, terminology, etc., that is formidable in size and scope. Several high-level IBM managers have been appointed to routinely visit centers of UXD in order to assess the quality and consistency of attention to best practices. One of them, Karel Vredenburg, has even published a book about their approach (Vredenburg et al, 2002).

In Europe, SAP has established the SAP Design Guild, which brings together a large body of information about best practices (http://www.sapdesignguild.org/). To its credit, this Website is open to all viewers/visitors, The SAP Design Guild has also published books that explain aspects of its views of best practices.

At HP, in approximately 2004, shortly before her reign as CEO of HP ended, according to anecdotes provided by HP UXD managers at the time, Ms. Carly Fiorina asked that the company Website announce a new initiative: that the company had made the "customer experience" a top priority. This signaled a significant shift in design, branding, product development, and more. During the years since then significant efforts have been made to overhaul human factors, industrial design, and interaction design teams and processes at HP. Interbrand reported in 2008 that HP (http://www.interbrand.com/portfolio_details.asp?portfolio=2564) undertook a major rebranding effort called "One Voice," which seeks "to better integrate its vast line of consumer electronics and computer hardware products. With a fresh design to the packaging, they faced a challenge to stay on brand across thousands of product lines and dozens of packaging types. This would inevitably require re-alignment of large 
teams of people, the establishment of templates and clear archives of canonical processes, procedures, roles, and definitions. In recent years, Mr. Sam Lucente was appointed Vice-President for Design at HP, an unusual ascension in the IT world of an executive-level person responsible for instituting superior design processes. For those interested, a YouTube 15-minute interview is available about the role of design at HP: (http://video.google.com/videoplay?docid=2924735257204340870).

Regarding AT\&T, in approximately, 2003, the author learned that there had been a "user-experience engineering" group at AT\&T since about 1997, or perhaps earlier. An article about that group appears in an article by Cunningham et al (2001). The abstract states: "The authors design and evaluate Internet-based applications as members of the User Experience Engineering Division of AT\&T Labs. They all started in academia with doctorates in cognitive psychology, and have been at AT\&T Labs for periods ranging from two to 17 years."

For about a decade, Microsoft has been buying up some of the best $\mathrm{R}+\mathrm{D}$ talent it can afford to strengthen Microsoft Research. Its general user-experience development teams for existing product development seem to have lagged, and the mixed results of its product deployment have been noted in news media and the Internet. Nevertheless, Microsoft made significant advances in the early 21 st century to hire anthropologists and ethnographers to join their UXD teams, so much so that a unit of Fortune magazine online named Bill Gates one of the great anthropologists of all time, somewhat tongue-in-cheek. The accolade did point to the significant ascension of anthropology and ethnography to the professionals required to achieve significant improvements in UXD.

Also of note is Microsoft's outreach to designers in its new product line and the MIX conferences, which seek to bring together software developers with designers, to raise the consciousness of both "tribes." Microsoft has appointed evangelists to communicate with the design communities. Another example of outreach and crossdisciplinary communication and interaction are the job outreach programs to design schools. A recent announcement (April 2008) from the Illinois Institute of Technology's Institute of Design, Chicago, is instructive:

"MS is footing the bill for drinks and appetizers, in exchange for Jakob Nielsen [not Jakob Nielsen of Nielsen Norman Group, but another person] doing a short pitch, as part of a national recruiting campaign for growing design, design research and UX at MS, which he heads up. ....You are invited to a special ID alumni reception, graciously sponsored by Microsoft. Free food and drink, old and new friends, plus two guests from Redmond: Jakob Nielsen, Director of User Experience, and Safiya Bhojawala (M.Des. '06), User Experience Researcher in the MS Dynamics User Experience Team..."

This kind of outreach to the design community is something that other enterprises should note and consider emulating if they wish to attract the kind of talented individuals required to generate breakthrough user experiences in the coming decades. These user experiences will require increased attention to "emotional design," "brand values," "aesthetics" and "customer culture", including issues of "identity", topics that are quite different from previous decades that focused on usability, i.e., effectiveness, efficiency, and satisfaction, according to the ISO definition. 


\section{Acknowledgments}

The author acknowledges the assistance Mr. Niranjan Krishnamurthi in the preparation of this paper.

\section{References}

1. Cockton, G.: Value-Centred HCI. In: Hyrskykari, A. (ed.) Proceedings, NordiCHI 2004, Tampere Finland, October 23-27, pp. 149-160 (2004)

2. Cunningham, J.P., Cantor, J., Pearsall, S.H., Richardson, K.H.: Industry Briefs: AT+T. Interactions 8(2), 27-31 (2001)

3. European Design Centre: User-Centred Design Works (CD-ROM). IOP Human Machine Interaction (2004) This CD-ROM presents a case for user-centered design including case studies and information resources, http: / / www. edc.nl

4. Gaddy, C., Marcus, A.: Analyze This: A Task Analysis Primer for Web Design. User Experience 5(1), 20-23 (2006)

5. Marcus, A.: Global/Intercultural User-Interface Design. In: Jacko, J., Spears, A. (eds.) Handbook of Human-Computer Interaction, 3rd edn., ch. 18, pp. 355-380. Lawrence Erlbaum Publishers, New York (2007)

6. Marcus, A.: What Would an Ideal CHI Education Look Like? Fast Forward Column, Interactions 12(5), 54-55 (2005)

7. Marcus, A.: Usability Grows Up: The Great Debate. Fast Forward Column, Interactions 12(4), 72-73 (2005),

http://www.uigarden.net/english/reviewsandinterviews.php/

2005/08/20/usability_grows_up_the_great_debate

(English),

http: //www. uigarden.net/chinese/reviewsandinterviews . php/ 2005/08/20/da_bian_lun (Chinese)

8. Marcus, A.: User-Centered Design in the Enterprise. Fast Forward Column, Interactions 13(1), 18-23 (2005)

9. Marcus, A.: User Interface Design's Return on Investment: Examples and Statistics. In: Bias, R.G., Mayhew, D.J. (eds.) Cost-Justifying Usability, 2nd edn., ch. 2, pp. 17-39. Elsevier, San Francisco (2005)

10. Schaeffer, E.: Institutionalization of Usability. Addison-Wesley, Reading (2004)

11. Vredenburg, K., Insensee, S., Righi, C.: User-Centered Design: An Integrated Approach. Software Quality Institute Series. Prentice Hall, Upper Saddle River (2002)

\section{URLs and Email Contacts}

The following URLs and email contacts, among others, are relevant to this topic:

- http://www-3.ibm.com/ibm/easy/eou_ext.nsf/publish/

- 1996

- http://www-2.cs.cmu.edu/ bam/uicourse/special/

- http://www.usabilitynet.org/tools/13407stds.htm

- http://www.usabilityprofessionals.org/upa_publications/ux_poster.html

- http://www.amanda.com/services/approach/approach_f.html

- http://www.usabilityprofessionals.org/usability_resources/guidelines_and_methods/ methodologies.html 
- http://www.effin.org/. EFFIN is a multi-client sponsored project to tailor usercentered methods to the development of electronic government services and to investigate these methods in practice. The project is financed through the FIFOS program of the Norwegian Research Council

- http://www.upassoc.org/upa_projects/usability_in_enterprise/index.html. The project, sponsored by the European usability community seeks to gather information on usability practice in the enterprise and evidence of return on investment (ROI) of usability. 